\title{
Photonics-based radar for sub-mm displacement sensing
}

\author{
Sergio Pinna, Suzanne Melo, Francesco Laghezza, Filippo Scotti, Emma Lazzeri, Mirco Scaffardi, \\ Paolo Ghelfi, Antonella Bogoni
}

\begin{abstract}
Photonics-based multiband radars have been demonstrated where photonics is exploited for multiple RF signal generation and detection by means of a single optical local oscillator that replaces the conventional cascades of electrical local oscillators. The ultra-wide band and high stability of photonics and the use of a single local oscillator assure very low system phase noise and phase coherence among the RF signals. This phase coherence among multi-band signals is exploited to perform differential phase estimation in enhanced sub-millimeter displacement measures. The system employs stepped frequency continuous waves simultaneously in the $S$ - and $X$-band, measuring the differential phase over a frequency span up to 7.4GHz. The high coherence among the two frequency bands, provided by the photonic architecture, enables very precise displacements measures, allowing to obtain sub-millimeter precision without using correction algorithms.

The presented experimental results demonstrate a precision $<200 \mu \mathrm{m}$ in a range up to $3 \mathrm{~km}$. Moreover, the sharing of the same hardware to handle a multi-band operation allows a great reduction of size, weight, power and footprint of the overall system.
\end{abstract}

Index Terms-differential phase estimation, dual-band radar. microwave photonics, photonic transceiver.

\section{INTRODUCTION}

$\mathrm{R}$ eal-time monitoring of sub-millimeter displacements is a useful task in more and more applications related to the environment monitoring (displacements of ground or big structures) [1], security (e.g., displacements of buildings) [2], or industrial processes (displacement of industrial machineries etc.) [3]. As a significant example, the monitoring of landslides is a key enabling strategy in order to prevent and reduce natural hazards produced by ground failures. They are responsible for great damages, as human suffering, huge economic losses and environmental degradation [4]. Over the

Manuscript received June 1, 2016. This work was supported in part by the EU projects RAPIDO (\#619806), ROAM (\#645361), FiWin5G (\#642355), and PETRA (\#641388).

Sergio Pinna, Suzanne Melo, Emma Lazzeri and Antonella Bogoni are with the Scuola Superiore Sant'Anna, Via G. Moruzzi 156124 Pisa, Italy (email: s.pinna@sssup.it)

Francesco Laghezza, Filippo Scotti, Mirco Scaffardi and Paolo Ghelfi are with CNIT (Consorzio Nazionale Interuniversitario per le Telecomunicazioni) Via G. Moruzzi 156124 Pisa, Italy. years, many different technologies were employed in order to map and control the movement of ground displacements as extensometers [5], crack meters [6], inclinometers [7], laser or sonar range finders [8], GPS receivers [9], etc. The main limitation associated with all these solutions is the inability to provide a deformation information on large areas, being used only for localized solutions. Moreover, their installation can be difficult, expensive and time consuming in order to be effective, especially when applied to monitoring of broad and inaccessible areas [10]. An alternative and attractive solution is the use of a remote sensing approach based on radars, capable of overcoming the limitations already mentioned, and remotely providing a complete and precise deformation map of the whole area under surveillance [11-14].

In the recent years, radars for retrieving ground displacements were developed and demonstrated to achieve very high resolution and long-range capabilities. The commercially available radar systems are based on single-band differential interferometry [14], with the technique of steppedfrequency continuous wave (SFCW) [15]. The SFCW technique consists on the use of several coherent sine waves in a single RF band to illuminate and detect the positions of different targets located along the radar line of sight. Through differential interferometry, the collected echoes are processed by employing differential phase algorithms, which translates the phase variations into small displacement measures. The combination of several sinusoidal waveforms allows to synthesize a large signal bandwidth, which provides improved range cell resolution (i.e. the minimum distance among two targets to be detected as independent targets), and reduction of noise for an enhanced differential phase estimation. An important parameter that has a great impact on the phase estimation precision is the coherence among the sinusoidal waveforms. When there is a high coherence, undesirable phase fluctuations between two consecutive observations are minimized, and for this reason, the measured phase variations will correspond entirely to the range shift of interest. In this way, a high coherence can lead to very precise displacements measures.

Nevertheless, it would be highly desirable to have a system operating in a coherent multi-band configuration. This feature would strong benefit the displacement measure accuracy, due to the possibility of applying the advanced interferometric techniques among the different frequency 

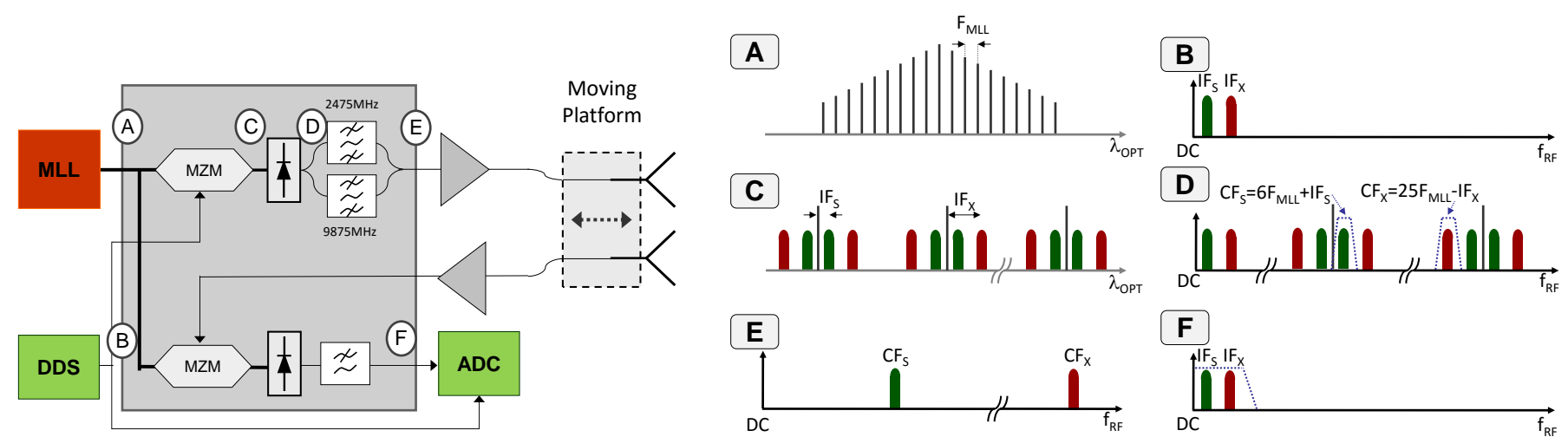

Fig. 1 - (left) Scheme of the photonics-based displacement measure experiment; (right) insets of the spectrum in the highlighted points. (A) optical spectrum of the MLL; (B) electrical spectrum of the applied signals; (C) optical spectrum of the modulated signals after the MZM; (D) electrical spectrum at the photodiode's output, with the following RF filters highlighted; $€$ electrical spectrum of the filtered RF signals before the wideband amplifier; (F) electrical spectrum of the received signal after the receiver $\mathrm{PD}$ and after the low-pass filter.

bands and allowing to tune the operative RF carriers for adapting the system to the environment (as weather condition or observed scenario) and to the range of interest [16]. The combination of these characteristics would greatly enhance the final accuracy in performing differential phase measurements contributing to a reliable early risk detection.

However, the generation of multi-band signal over a wide frequency range by actual electronic systems is a challenging task, which greatly increase system complexity and cost [17].

Recently, photonics technologies have been introduced in the microwave field in order to overcome many of the limitations of electronics. Photonics has been demonstrated for an efficient generation and detection of several simultaneous coherent high-frequency radiofrequency (RF) signals. In 2013 and 2014 the first photonics-based coherent single-band [18] and dual-band [19] radar system were demonstrated respectively. In [19] the system exploits a single highly stable optical clock as optical local oscillator for generating and detecting (through photonic up- and down conversion of the waveforms) all the RF signals with an intrinsic high phase coherence among the carriers, allowing coherent multi-band radar operations.

In this work we take advantage of the novel photonics-based multiband radar system in order to perform precise displacement measures. The possibility of exploiting more than one different band confers improved resolution to the system, which combined with the huge bandwidth and flexibility of photonics allow to carry out an extended analysis based on SFCW and differential interferometry. The obtained flexibility refers to the system's capacity of dynamically tune the RF operative carrier in a software defined manner. The high phase coherence among the RF carriers allows to reduce the complexity, or avoids the use of correction algorithms. Finally, a great reduction of the overall system size, weight and power consumption (SWaP) is obtained through the use of a single transceiver to handle a multi-band approach.

This work reports for the first time, for the best of our knowledge, the system characterization and the experimental results of small displacement measures based on SFCW technique and differential interferometry using a photonicsbased dual-band coherent radar operating in S- and X-bands. The proposed system suitable for the monitoring of landslides or civil structure (buildings, dams, ect.) demonstrates the capability of the proposed architecture to reach displacement accuracy in the sub-millimeter scale, with a measure error lower than < $200 \mu \mathrm{m}$ for targets over a range up to $3 \mathrm{~km}$ without correction algorithms.

\section{OPERATING PRINCIPLE}

The radar exploits the combination of two different techniques to determine target displacement and position: SFCW and differential interferometry.

The target is illuminated by several sinusoidal signals, coherent to each other, with frequency $f_{\mathrm{n}}$ and separation of $\Delta f$, and the displacement monitoring is based on differential phase measurements. Each frequency component of the backscattered echo accumulates a different phase shift $\varphi_{\mathrm{n}}$, depending on the traveled distance $d$ :

$$
\varphi_{n}=2 \pi \cdot f_{n} \cdot \frac{2 d}{c}
$$

Thus, for a given range, two frequency separated by $\Delta f$ will present a relative phase difference $\Phi$ :

$$
\Phi=2 \pi \cdot \Delta f \cdot \frac{2 d}{c}
$$

so that any change $\Delta d$ in the distance induces a phase variation $\Delta \Phi$, related by the following relationship:

$$
\Delta d=\frac{c}{4 \pi \cdot \Delta f} \cdot \Delta \Phi
$$

If the phase varies more than $2 \pi$, ambiguities come out. In fact this kind of technique presents an unambiguous range given by: 


$$
R_{u}=\frac{c}{2 \Delta f}
$$

Taking into account (3), it's worth to notice that a given precision in the phase estimation corresponds to a different accuracy in the range determination depending on $\Delta f$.

The standard deviation on the phase estimation is given by [20]:

$$
\sigma_{\Phi}=\frac{1}{\sqrt{S N R}}
$$

Where SNR is the Signal-to-Noise Ratio of the received signal.

As the phase estimation accuracy is independent on the signal frequency, for a given SNR the higher is the frequency difference $\Delta f$ more precise is the distance measure (eq. 3), at the cost of reducing the unambiguous range (see eq. 4) [21]. In this way, the use of high $\Delta f$ makes possible to detect displacements much smaller than a fraction of millimeter [21].

Furthermore, if $N$ is the number of sinusoids forming the SFCW signal, the total signal bandwidth $B W$ is given by:

$$
B W=(N-1) \cdot \Delta f
$$

As for a radar system, the range cell resolution $\Delta R$, achievable by applying standard radar processing algorithms on the received signal, is given by the transmitted signal bandwidth:

$$
\Delta R=\frac{c}{2 \cdot B W}=\frac{c}{2 \cdot(N-1)} \cdot \Delta f
$$

This way the same SFCW signal can be exploited for both rough positioning of the target and simultaneous displacement measure, even allowing the system to recognize and monitor multiple targets at different distance within the antenna beam width.

To this aim, the photonic transceiver, guaranteeing the coherence between different RF bands, is able to provide an extremely high synthetic frequency, i.e., the combination of $\mathrm{N}$ individual sinusoidal tones in order to synthesize a larger bandwidth. The high synthetic frequency combined with the low phase noise characteristics, allow a precise differential phase estimation, which, as already stated, can be translated into very small displacement measures.

\section{PHOtONICS-BASEd RADAR SYSTEM FOR DIFFERENTIAL PHASE MEASUREMENTS}

The scheme of principle of the multi-band photonics-based radar system used to perform differential phase measurements is depicted in Fig. 1. The key element of the system is a modelocked laser (MLL) which acts as optical local oscillator and allows to perform coherent up- and down-conversion of the RF signals. In the up-conversion, the MLL generates an extremely precise optical pulse train (Fig. 1A) with a repetition rate of $\mathrm{F}_{\mathrm{MLL}}=400 \mathrm{MHz}$. The optical modes are then modulated via a Mach-Zehnder modulator (MZM) by a direct digital synthesizer (DDS), generating two different SFCW waveforms at two different intermediate frequencies (IFs), respectively $\mathrm{IF}_{\mathrm{S}}=75 \mathrm{MHz}$ and $\mathrm{IF}_{\mathrm{X}}=125 \mathrm{MHz}$, as shown in Fig. $1 \mathrm{~B}$ (S and $\mathrm{X}$ are referred to the frequency bands of the up-converted transmitted signal). By means of the MZM, the waveforms are then transferred as lower and upper sidebands around each optical mode of the MLL (Fig. 1C). In this way the IF spectrum at the input of the MZM, is replicated, in the optical domain, every $\mathrm{F}_{\mathrm{MLL}}$. In order to avoid aliasing, the sum of the intermediate frequencies and their spectral occupancy must be less or equal the half of the MLL repetition rate. After the MZM, a photodiode (PD) is used to perform the heterodyning of all spectral components, which will produce RF signal replicas at carrier frequencies $\mathrm{CF}_{\mathrm{S}}=\mathrm{k} \cdot \mathrm{F}_{\mathrm{MLL}} \pm \mathrm{IF}_{\mathrm{S}}$ and $\mathrm{CF}_{\mathrm{X}}=\mathrm{m} \cdot \mathrm{F}_{\mathrm{MLL}} \pm \mathrm{IF}_{\mathrm{X}}$, respectively (Fig. 1D), with $\mathrm{k}$ and $\mathrm{m}$ positive integers. Then, it is possible to select the required upconverted signals at $\mathrm{CF}_{\mathrm{S}}=2475 \mathrm{MHz}$ (S-band) and $\mathrm{CF}_{\mathrm{X}}=9875 \mathrm{MHz}$ (X-band) by using two electrical band-pass filters (BPF), one for each desired band (Fig. 1E). In this way, the carrier frequencies can be selected independently among the several generated replicas. A wideband RF amplifier (WBA) at the end of the transmitter side will boost the RF signal, which is going to be transmitted by a Horn antenna.

At the receiver side, a second identical antenna collects the $\mathrm{RF}$ echo which is going to be amplified by another WBA. The $\mathrm{RF}$ received signal is transferred to the optical domain through another MZM that modulates the same optical pulse train provided by the MLL. This modulation generates replicas of the received RF waveform around each of the MLL modes at RF spectral distance. The obtained optical spectrum is analog to the spectrum in Fig. 1C. Then, the heterodyning in another PD of each replica with the closest optical carrier, produces a down-converted replica of the received RF carriers in the original IF spectral region. The down-converted signal is then filtered by an electrical low-pass filter (Fig. 1F) and sent to a 400MSample/s analog-to-digital converter (ADC), which digitizes the signal and performs the required processing. The ADC also digitizes a copy of the signal generated by the DDS in order to obtain phase reference. The coherence is guaranteed by the MLL, which serves as a reference for both up- and down-conversion of the radar signal.

\section{EXPERIMENT}

Two broadband horn antennas with 60 degree beam width, one for signal transmission and one for the backscattered signal collection, were placed on a digitally controlled motorized linear platform (Fig.2). The motorized slide, remotely controlled, allows a precise antenna positioning. Indeed, due to practical limitations, the displacements measurements were carried out by moving the antenna assembly, rather than the target, and using the laboratory wall, in the antenna far field region, as a target. The reference location, used for the displacement measure, was set to the slide central position, where the rail has been first positioned, thus allowing to set both positive and negative target 
displacements.

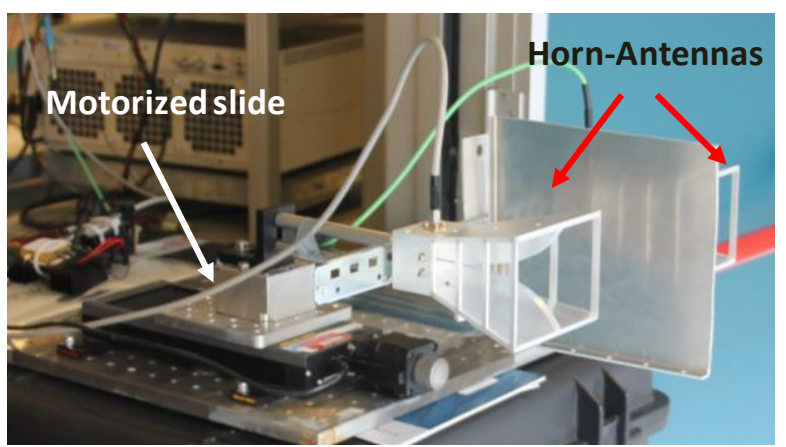

Fig. 2 - Moving antenna setup

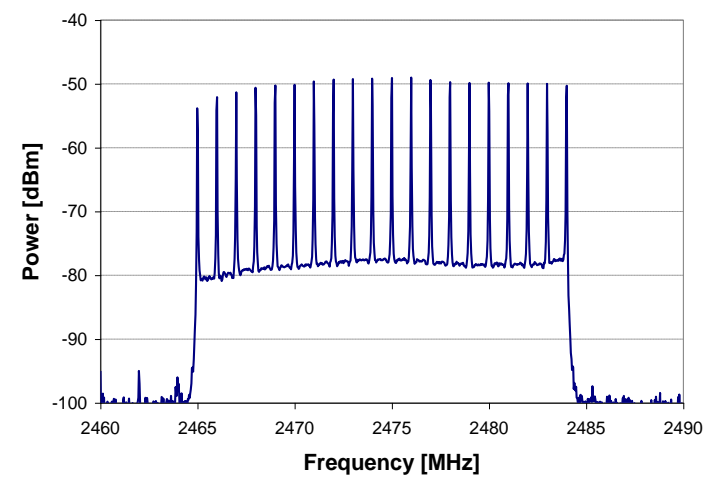

A

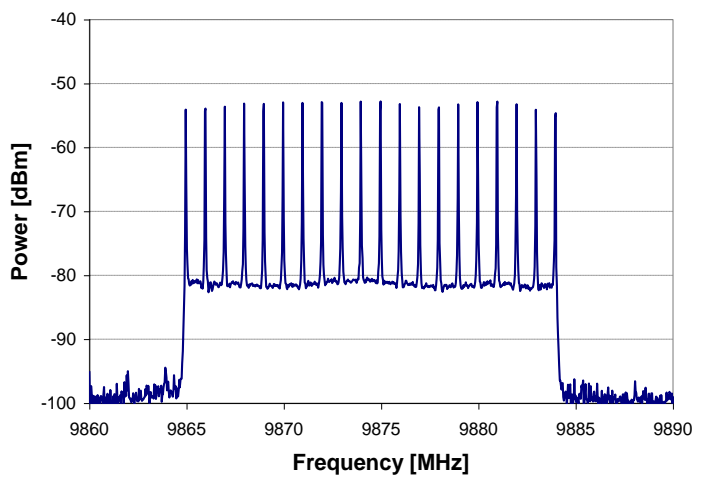

B

Fig. 3 - Electrical spectrum of the dual-band RF signal a) S-band signal; b) X-band signal

The transmitted signal, which spectrum is reported in Fig. 3 is formed by two SFCW, each one composed by 20 frequency steps (limited by the available RF filter bandwidth), generated at their intermediate frequency, respectively of $75 \mathrm{MHz}$ and $125 \mathrm{MHz}$. Each step has a duration of $200 \mu \mathrm{s}$ and they are equally spaced by $1 \mathrm{MHz}$. The signal is then up-converted at RF by the photonics-based transceiver at, respectively, the Sband (2.475GHz, Fig. 3A) and X-band (9.875GHz, Fig.3B). The S-band and $\mathrm{X}$-band signals are then coupled and amplified by means of a broadband electrical amplifier and then radiated by the transmitting antenna.

At the receiver side, the signal scattered by the target is collected by the second horn antenna, amplified by a low noise broadband amplifier and received by the photonic transceiver, where the signal is down-converted back to IF to be digitized and processed.

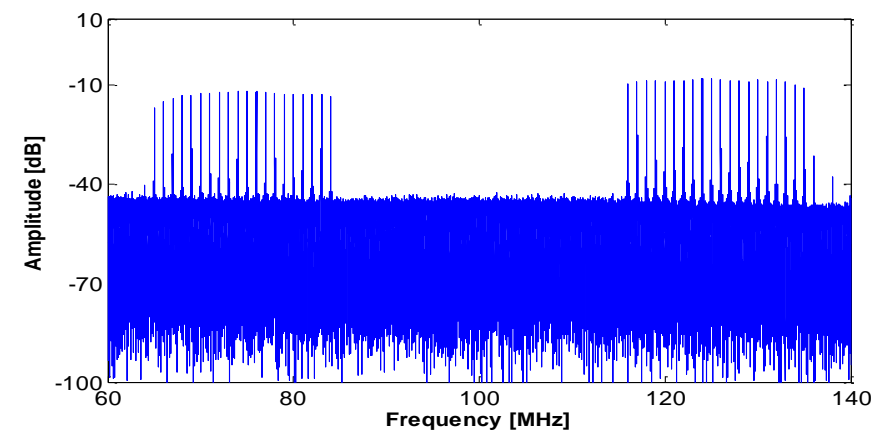

Fig. 4 - IF-spectrum of the received SFCW signal.

In order to evaluate the target displacement, the amplitude and phase spectrum of the received down-converted echo (Fig. 4) and the reference signal (i.e., a copy of the transmitted signal Fig.5) has been first evaluated via standard fast Fourier Transform (FFT). The phase of the 40 SFCW tones was then extracted after multiplying together the echo spectrum and the complex conjugate of the reference one. Finally, applying eq. (3) at the phase difference among the S and X-band SFCW tones leads to the actual target displacement.

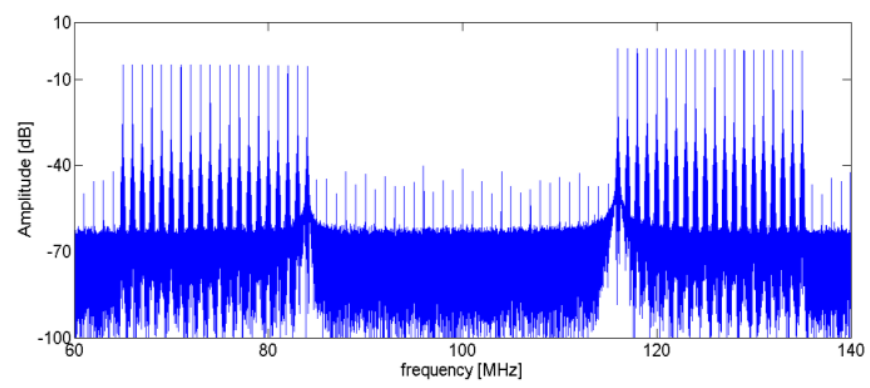

Fig. 5 - IF-spectrum of the generated SFCW signal

As the total bandwidth of the SFCW signal is $40 \mathrm{MHz}$ the range cell resolution $\Delta R$ obtained by applying standard radar processing algorithms on the received signal is equal to $\Delta \mathrm{R}=$ $3.25 \mathrm{~m}$.

Furthermore, using the differential phase technique, the minimum frequency step $\Delta f$ of $1 \mathrm{MHz}$, is able to guarantee a maximum unambiguous distance is of about $300 \mathrm{~m}$, however, higher displacement resolution are obtained exploiting the dual band operation, making possible to obtain 20 independent measures with a maximum frequency difference $\Delta f$ up to $7.4 \mathrm{GHz}$, leading to a minimum unambiguous range of $2 \mathrm{~cm}$. 

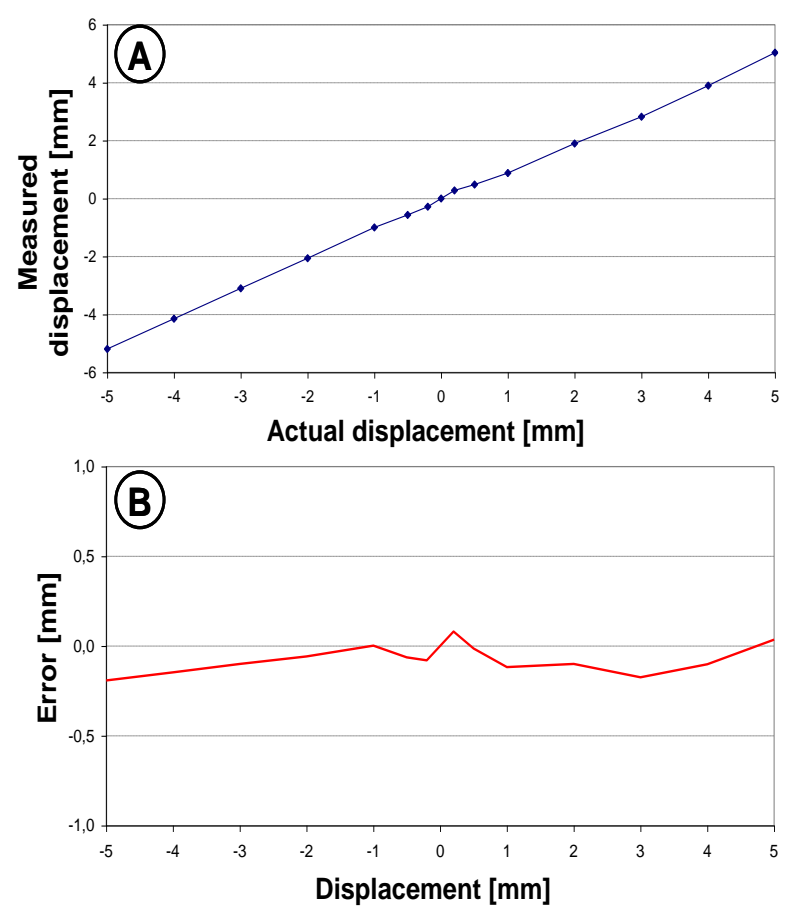

Fig. 6. A) Measured displacement vs. real displacement; B) Displacement measure error.

\section{RESULTS}

To simulate the target displacement, the antenna position is moved by steps as small as $0.2 \mathrm{~mm}$ and with a $10 \mathrm{~mm}$-long total excursion (limited by the motorized slide), with the target (the laboratory wall) about two meters apart from the radiating elements.

For each step a radar acquisition is made and compared with the reference position which, in this case, was the centre of the motorized rail, thus providing the target displacement.

In Fig. 6-A, the measured displacement (y-axis), as a function of the actual stage position (x-axis) is reported. The measurement, results to be in a good agreement with the real target position, with a maximum measurement error Fig. 6-B, limited to less than $\pm 0.2 \mathrm{~mm}$.

As in a real application, such as mine or structural monitoring, the target could be located up to few $\mathrm{km}$ away from the radar, the measure has been repeated for different target distances. Since one of the sources of error in the phase difference estimation is determined by the intrinsic phase noise of the radar system, adding a delay between the signal generation and reception decorrelates the echo and the reference signal, thus providing a better estimation of the system performance.

To this aim, several target distances has been simulated by adding, respectively, one and two $1 \mathrm{~km}$-long optical fiber spools in the radar transceiver as delays. Since the refractive index of optical fibers is about 1.5, such fiber spans emulate, respectively, a distance of $1.5 \mathrm{~km}$ and $3 \mathrm{~km}$.

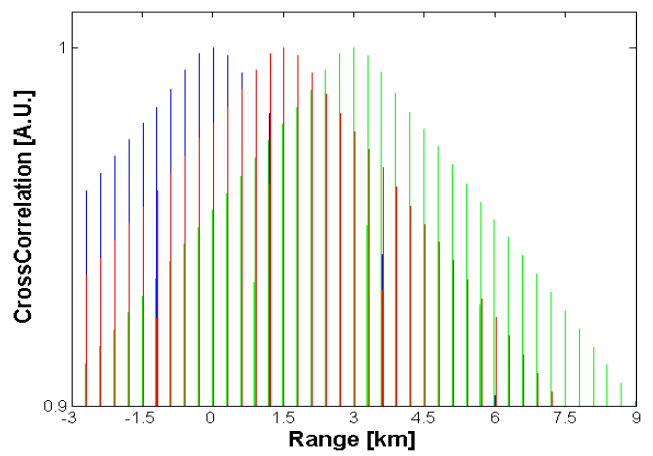

Fig. 7. Experimental cross-correlation function of the received echo for $0 \mathrm{~km}$ (blue), $1.5 \mathrm{~km}$ (red) and $3 \mathrm{~km}$ (green) distant targets.

The range cell, i.e. the target distance, has been measured by evaluating the peak of cross-correlation function between the collected echo and the reference signal. As shown in Fig. 7, in which the blue plot reports the $0 \mathrm{~km}$ case, the red plot the $1.5 \mathrm{~km}$ target and the green one the $3 \mathrm{~km}$ target, the measured ranges perfectly match with the nominal length of the fiber spools.

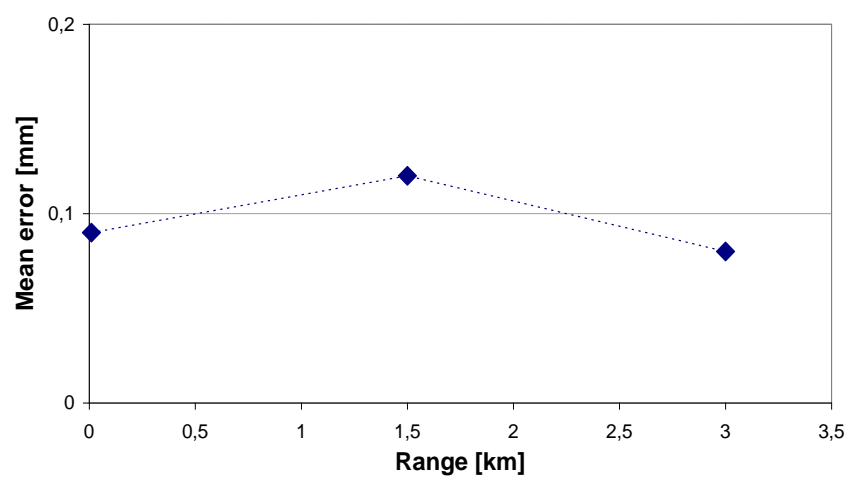

Fig. 8. Mean error of the displacement measurement error.

As can be seen in Fig. 8, reporting the mean value of absolute displacement error at different ranges, the error remains almost constant among the $3 \mathrm{~km}$ target distance and below $0.2 \mathrm{~mm}$, thus confirming that the phase stability of the developed radar system is suitable for phase interferometry techniques.

Another limiting factor in the phase estimation is given by the received signal SNR. Indeed, as expressed by eq. (4), for a given SNR the minimum standard deviation of the phase estimation is determined.

In Fig.9 the displacement precision, expressed in millimeters is reported as a function of the signal SNR. The green curve reports the theoretical displacement precision for a single $7.4 \mathrm{GHz}$ sinusoidal signal, equal to the frequency difference among the $\mathrm{S}$ and $\mathrm{X}$ bands. Such curve represent the maximum accuracy achievable by the if system transmitting a 


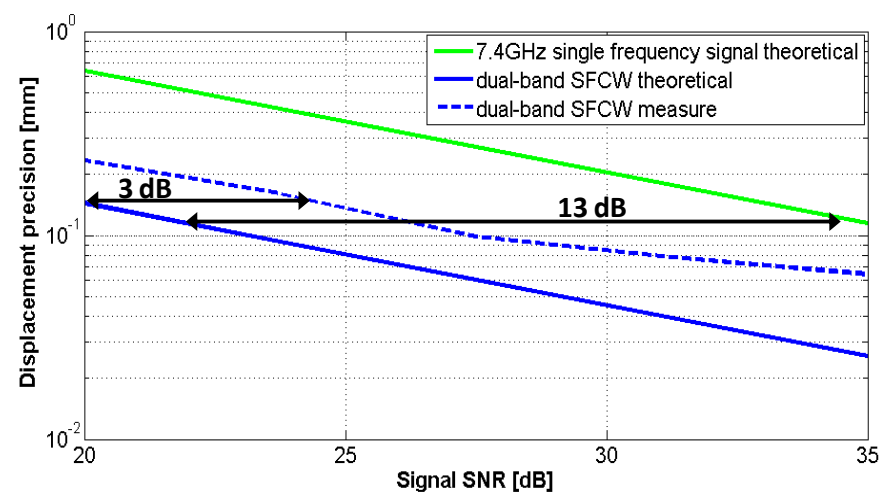

Fig. 9 Dislacement precision vs signal SNR.

single sinusoidal tone for each of the two bands rather than the SFCW signal. Instead, the blue lines, show the displacement precision achieved by means SFCW signal of 20 steps $(20$ sinusoidal signal for each frequency band), in the ideal case (blue solid line, simulated result) and the measured one (dotted line).

Comparing the theoretical results it is possible to notice that the SFCW curve present about $13 \mathrm{~dB}$ SNR gain compared to the single frequency case. This gain is ascribed at the averaging gain. Indeed, as $N$ independent phase measurements are coherently averaged, the variance of the estimated parameter decreases (and consequently the SNR increases) by a factor of $N$. In this case, as $N=20$, thus leading to an SNR gain, induced by the averaging, equal to $13 \mathrm{~dB}$.

Instead, the dashed line correspond to the system measure error. Compared to the theoretical one, it is possible to notice $3 \mathrm{~dB}$ loss in the low SNR region, while above $27 \mathrm{~dB}$ of SNR is even possible to notice a decrease of the curve slope, indicating that for high values of signal SNR, the improvements in the phase estimation are reduced, since effects of system saturation (e.g., amplifiers, ADCs, ect.) take place, becoming impossible to reach the theoretical value.

\section{CONCLUSION}

This work reports for the first time, to the best of our knowledge, a photonics-based radar system exploiting multiband stepped-frequency continuous wave (SFCW) signals with differential interferometry to perform displacement measurements.

The use of photonics allows for the generation and detection of tunable and multi-band RF signals with intrinsic high phase coherence that allows to obtain sub-mm resolution without correction errors.

In the proposed experiment the photonics-based radar transceiver exploits a mode locked laser to generate 40 coherent sinusoidal waveforms distributed in S- and X-bands, synthesizing a band of $7.4 \mathrm{GHz}$.

Experimental results demonstrate an accuracy $<200 \mu \mathrm{m}$ for displacement measurements up to $3 \mathrm{~km}$ of distance.

The use of a single photonics-based transceiver for handling the signals in both the RF bands allows for a reduction of the size, weight, power consumption and footprint of the whole system.

\section{ACKNOWLEDGMENT}

This work has been supported in part by the EU projects RAPIDO (\#619806), ROAM (\#645361), FiWin5G (\#642355), and PETRA (\#641388).

\section{REFERENCES}

[1] D. Tarchi, et al., "Monitoring landslide displacements by using groundbased synthetic aperture radar interferometry: Application to the Ruinon landslide in the Italian Alps", Journal of Geophysical Research, vol. 108 , issue $\mathrm{B} 8,2003$.

[2] M. Pieraccini, "Monitoring of Civil Infrastructures by Interferometric Radar: A Review", The Scientific World Journal, v. 2013, 2013.

[3] G. Vinci, et al., "Six-Port microwave interferometer radar for mechanical vibration analysis", In proceeding of the European Radar Conference (EuRAD), pp. 287-290, Nuremberg, 2013.

[4] G. P. L. Spiker E.C., "National landslide hazards mitigation Strategy - A framework for loss reduction," US Geol. Surv. Circ., no. 1244, pp. 154, 2003.

[5] 5. J. Corominas et al., "Measurement of landslide displacements using a wire extensometer", Engineering Geology vol. 55, no 3, pp. 149-166, 2000.

[6] 6. M. J. Kavvadas, "Monitoring ground deformation in tunnelling: Current practice in transportation tunnels", Engineering Geology, v. 79, no 1-2, pp.93-113, 2005.

[7] 7. X. Hou, X. Yang, and Q. Huang, "Using Inclinometers to Measure Bridge Deflection", Journal of Bridge Engineering, vol. 10, $\mathrm{n}^{\mathrm{o}} 5$, pp. 564-569, 2005

[8] 8. M-C. Amann, T. Bosch, M. Lescure, R. Myllyla, and M. Rioux, "Laser ranging: a critical review of usual techniques for distance measurement", Optical Engineering, v. 40, $\mathrm{n}^{\circ} 1$, pp. 10-19, 2001.

[9] 9. B. W. Crowell, Y. Bock, and M. S. Squibb, "Demonstration of Earthquake Early Warning Using Total Displacement Waveforms from Real-time GPS Networks", vol. $80 \mathrm{n}^{\circ}$ 5, 2009.

[10] W. H. Schulz, J. a Coe, B. L. Shurtleff, J. Panosky, P. Farina, P. P. Ricci, and G. Barsacchi, "Kinematics of the Slumgullion landslide revealed by ground-based InSAR surveys," pp. 1273-1279, 2012.

[11] G. Antonello, N. Casagli, P. Farina, D. Leva, G. Nico, a. J. Sieber, and D. Tarchi, "Ground-based SAR interferometry for monitoring mass movements," Landslides, vol. 1, no. 1, pp. 21-28, 2004.

[12] N. Casagli, F. Catani, C. Del Ventisette, and G. Luzi, "Monitoring, prediction, and early warning using ground-based radar interferometry," Landslides, vol. 7, no. 3, pp. 291-301, 2010.

[13] D. Tarchi, "Monitoring landslide displacements by using ground-based synthetic aperture radar interferometry: Application to the Ruinon landslide in the Italian Alps," J. Geophys. Res., vol. 108, no. B8, p. 2387, 2003.

[14] M. Marchisio, L. Piroddi, G. Ranieri, S. V Calcina, and P. Farina, "Comparison of natural and artificial forcing to study the dynamic behaviour of bell towers in low wind context by means of ground-based radar interferometry: the case of the Leaning Tower in Pisa," J. Geophys. Eng., vol. 11, no. 5, p. 055004, 2014.

[15] 15. I. Nicolaescu, P. van Genderen, K. W. Van Dongen, J. Van Heijenoort, "Stepped frequency continuous wave radar-data preprocessing", in Proceedings of Advanced Ground Penetrating Radar, pp. 177-182, Delft, Netherlands, 2003.

[16] James, D. Taylor, and P. E. Taylor. "Ultra-wideband radar technology." CRC, London (2001).

[17] A. G. Huizing, "Wideband vs. multiband trade-offs for a scalable multifunction RF system", in Proceedings of IEEE International Radar Conference, pp. 155-160, 2005.

[18] P. Ghelfi, F. Laghezza, F. Scotti, G. Serafino, A. Capria, S. Pinna, D. Onori, C. Porzi, M. Scaffardi, A. Malacarne, V. Vercesi, E. Lazzeri, F. Berizzi, and A. Bogoni, "A fully photonics-based coherent radar system.," Nature, vol. 507, no. 7492, pp. 341-5, 2014.

[19] F. Scotti, F. Laghezza, P. Ghelfi, and A. Bogoni., "Multi-band softwaredefined coherent radar based on a single photonic transceiver," 
Microwave Theory and Techniques, IEEE Transactions on, vol. 63, no.2, pp.546-552, 2015.

[20] E. Rodriguez, and J. M. Martin, "Theory and design of interferometric synthetic aperture radars", IEEE Radar and Signal Processing, v. 139, ${ }^{\circ}$ 2, pp. 147-159, 1992.

[21] K. Meiners-Hagen, R. Schödel, F. Pollinger, and A. Abou-Zeid, "MultiWavelength Interferometry for Length Measurements Using Diode Lasers", Measurement Science Review, v. 9, nº1, 2009.

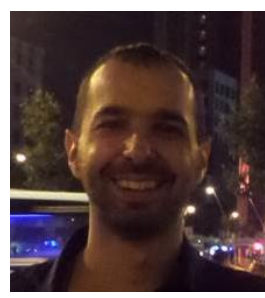

Sergio Pinna, received a $\mathrm{PhD}$ in Innovative Technologies from Scuola Superiore Sant'Anna, Pisa, Italy, in 2014, the B.Sc. and M.Sc. degree in telecommunications engineering from University of Pisa, Pisa, Italy in 2008 and 2010 respectively. From November 2010 to December 2015 he was fellow with CNIT at the National Photonic Networks Laboratory in Pisa.

In 2014 he was visiting researcher at Integrated Photonics Laboratory at Boston University, Boston (MA). Since 2016 he is research fellow at TeCIP Institute of Scuola Superiore Sant'Anna, Pisa, Italy. His research activity are mainly focused in the areas of microwave photonics for communication and remote sensing, all-optical signal processing and microwave photonic integration.

$\mathrm{He}$ is author and coauthor of 3 patents and 38 among international journal and international conference papers.

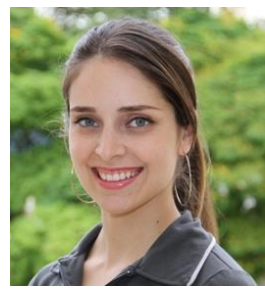

Suzanne A. S. Melo, was born in Barbacena, Brazil in 1989. She received her B.Sc.and M.Sc. degree in Telecommunications in 2012 and 2014 respectively from the National Telecommunications Institute (Inatel), Brazil. From April 2014 to October 2014 she was researcher for the National Interuniversity Consortium for Telecommunications (CNIT), Italy. Since November 2014 she is a $\mathrm{PhD}$ student at the TeCIP Institute of Scuola Superiore Sant'Anna of Pisa under the supervision of Dr. Antonella Bogoni. Her research activities are mainly focused on microwave photonics for radar systems and sensing.

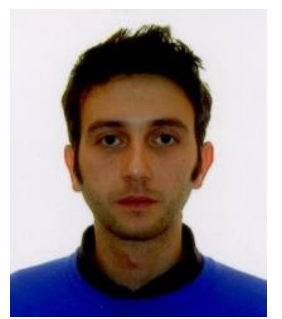

Francesco Laghezza, received the Master degree in Telecommunication Engineering and the Ph.D. degree in Remote Sensing from the University of Pisa (Italy) respectively in 2009 and 2013. From 2009 to 2010 he was a postgraduate research assistant for the National Interuniversity Consortium for Telecommunications (CNIT) working on a space debris ground radar system design for a Space Situational Awareness Network, and array processing for passive radars. From 2010 to 2013 he was a consultant within the CNIT working in several national and international research projects. He was technical leader for the radar system developing and prototyping group within the European projects PHODIR, PREPARE and PETRA. He is co-author of more than 70 papers in international peer-reviewed journals and conferences, and 5 patents. During his activities, he was honoured with the Young Engineering prize, during the 2014 International Radar Symposium, and with the Thales prize for his contribution in developing the first hybrid, LIDARIRADAR integrated architecture. Actually he is a Researcher at the CNIT working on a Microwave-Photonic Dual Band Radar (DBR) and fiber based distributed RADARCOM network, a photonic receiver for Electronic Spectrum Measurement (ESM) and SIGnal INTelligence (SIGINT) applications and also on LIDAR systems. His main activities are in the area of research and development of Radar systems with seven years' experience in system design, system engineering hands-on capability, including system and structural design, field test and calibration.

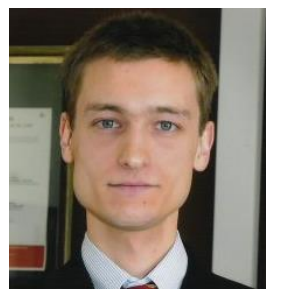

Filippo Scotti, was born in Milano, Italy in 1985. $\mathrm{He}$ received the Bachelor's degree in physics engineering in September 2007 and the M.S. degree in physics engineering in December 2009 from the Politecnico of Milano, Italy. In 2009 he was with PGT-Photonics, Milano for a six-month internship.

Since 2010 he is with the National Photonic Networks Laboratory of CNIT (Consorzio Nazionale Interuniversitario per le Telecomunicazioni), in Pisa, Italy. He authored or co-authored more than 50 papers in international journals and conferences. His research activity is in the area of fiber optic transmissions with particular interest in all-optical signal processing, microwave photonics, ultra-short optical pulse sources and their RF applications.

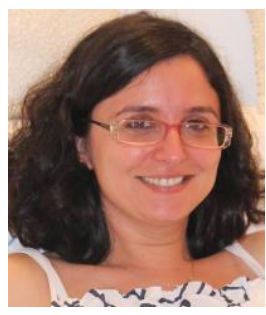

Emma Lazzeri was born in La Spezia, Italy, in 1981. She received the $\mathrm{PhD}$ in Innovative Technologies, Telecommunications from Scuola Superiore Sant'Anna, Pisa, Italy in 2013, and the Doctoral degree and the Magistralis Doctor degree in telecommunication engineering from the University of Pisa, Pisa, Italy, in 2004 and 2006, respectively. She was Visiting Researcher at NICT, Tokyo, Japan in 2008 and 2009 and at INRS, Montreal, Canada in 2009. Since 2010 She is a Research fellow at TeCIP, Scuola Superiore Sant'Anna, Pisa, Italy working in the Digital and Microwave Photonics group. Her research interest span from all-optical digital processing to microwave photonics. She is author and coauthor of 2 book chapters, 3 patents, 16 journals, and 25 International conference papers.

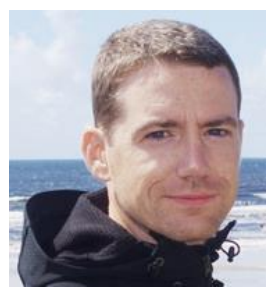

Mirco Scaffardi received the M.S. degree in electronics engineering from University of Parma, Parma, Italy in 2001. In 2005 he received the Ph.D. degree from the Scuola Superiore Sant'Anna, Pisa, Italy. He was Visiting Student at COM-DTU, Copenhagen, Denmark in 2004, and visiting researcher at NICT Tokyo, Japan in 2007 and 2008. In 2009 he was visiting researcher at INRS, Montreal, Canada awarded with a scholarship from state of Quebec. From 2011 he is Lecturer within Ph.D and international master courses of Scuola Superiore Sant'Anna. He is currently Senior Researcher with CNIT, Pisa, Italy.

$\mathrm{He}$ is Research Unit Coordinator of the Project "MIcro- and NanO-structured photonic devices based on strained silicon for ultrafast Switching in datacom applications (MINOS)" (2012-2014), FIRB, funded by the Italian Ministry of Education, University and Research and work package coordinator of the EU project ROAM.

$\mathrm{He}$ is co-author of more than 40 papers on refereed journal, 64 papers on referred conferences, 15 patent proposals and 2 book chapters. His research interests are in the area of all-optical signal processing, of photonic digital processing and ultrafast optical systems.

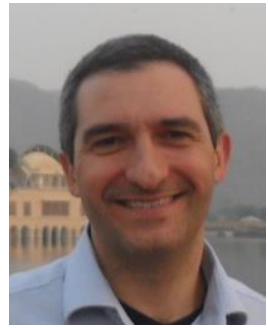

photonics.

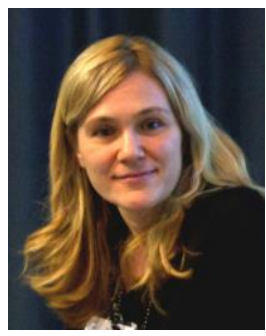

Antonella Bogoni, Professor at Scuola Superiore Sant'anna former head of research area of CNIT (National Inter-University Consortium for Telecommunications), is one of the pioneers of the Integrated Research Center for Photonic Networks and Technologies created in Pisa in 2001 as a part of a broad agreement among four partners, CNIT, Scuola Superiore Sant'Anna, Marconi Communications S.p.A. (now Ericsson) and the National Research Council of Italy (CNR), aiming to create in Pisa a world-class Center of Excellence, where currently she is leader of the "digital \& microwave photonics" area. 
Antonella Bogoni dedicated her research activity to photonics technologies for ultra-fast optical communication and microwave systems. In 2004 she proposed the fastest optical regenerator at that time, working at $320 \mathrm{~Gb} / \mathrm{s}$.

From 2008 she collaborates with the University of Southern California (USC) in Los Angeles including in 2009 as winner of a "Fulbright" scholarship. In the USC laboratories she developed the first photonic processor prototype suitable for logic operations at $640 \mathrm{~Gb} / \mathrm{s}$.

In 2009 she obtained an ERC starting grant for developing a photonic-based fully digital radar systems, presented on NATURE on 2014, and in 2012 and 2014 she got two additional ERC grants within the "proof of concept" program in order to convert her research results into pre-industrial products for airport security and environment monitoring. In 2015 she is coordinator of the H2020 project: "ROAM: Revolutionising optical fiber transmission and networking using the Orbital Angular Momentum of light"

Up to now Antonella Bogoni got 16 national and international project grants and 5 Best Paper awards. She is co/author of 55 patents, 8 books and chapters and more than 140 papers on the main scientific international journals (17 invited contributions).

She is Topical Editor for Optics Letters, she is also in the Board of reviewers of, Wiley Editor, Nature Photonics, IEEE/OSA Journals. She is chair of international conferences and workshops, including general chair of Photonics in Switching 2014, Sub-committee Chair in ECOC 2015 and Program Chair in ECOC 2018.

Her research interests are in the area of ultra-fast all optical signal processing high capacity interconnection networks, and microwave photonics, especially photonic-based RF signal generation and beam forming, analog-to-digital converters and electronic warfare receivers. 